\title{
E-PROCUREMENT: CONCEITOS, MODELOS E FATORES CRÍTICOS DE SUCESSO
}

\author{
Márcia Mazzeo Grande \\ mgrande@usp.br \\ Universidade de São Paulo - SP / Brasil \\ Enrico Arnaldo Olhê \\ Enrico.olhe@gmail.com \\ Universidade de São Paulo - SP / Brasil \\ Ildeberto Aparecido Rodello \\ rodello@fearp.usp.br \\ Universidade de São Paulo - SP / Brasil \\ Erasmo José Gomes \\ erasmo@ fearp.usp.br \\ Universidade de São Paulo - SP / Brasil
}

http://dx.doi.org/10.1590/1413-2311.06914.54830

Recebido em 13/04/2015

Aprovado em 25/07/2016

Disponibilizado em 31/01/2017

Avaliado pelo sistema "double blind review"

Revista Eletrônica de Administração

Editora-chefe: Aurora Zen

ISSN 1413-2311 (versão "on line")

Editada pela Escola de Administração da Universidade Federal do Rio Grande do Sul.

Periodicidade: Quadrimestral

Sistema requerido: Adobe Acrobat Reader

\section{E-PROCUREMENT: CONCEITOS, MODELOS E FATORES CRÍTICOS DE SUCESSO}

\section{RESUMO}

Este trabalho é uma revisão bibliográfica sobre o tema e-procurement e foi motivada pela importância crescente da atividade de compras para as empresas, pelo aumento do uso da tecnologia da informação na cadeia de suprimentos e pela escassez de trabalhos sobre o tema no Brasil. O objetivo foi compreender as práticas de e-procurement nas empresas, identificando os modelos existentes, as vantagens, benefícios, fatores críticos para a implementação e dificuldades de seu uso. Adicionalmente, foi realizado um balanço das publicações sobre o tema na coleção principal da Web of Science, indicando os períodos onde houveram mais publicações e também os periódicos que mais tem artigos relacionados. Assim, foi possível perceber as tendências sobre as pesquisas no assunto. Ao fím deste trabalho foi também possível chegar a conclusões importantes sobre a função estratégica do e-

REAd | Porto Alegre - Edição 85 - Nº 3 - Setembro / Dezembro 2016 - p. 312 - 335 
procurement, uma vez que modifica a maneira como as empresas se inserem na cadeia de suprimentos; o foco atual da tecnologia em compras de materiais indiretos, e uma série de benefícios não financeiros. O trabalho ainda contribui apresentando sugestões de assuntos para condução de pesquisas na área.

Palavras-Chave: E-procurement; Gestão da cadeia de suprimentos; Compras

\title{
E-PROCUREMENT: CONCEPTS, MODELS AND CRITICAL SUCCESS FACTORS
}

\begin{abstract}
This paper is a literature review on e-procurement and was motivated by the increasing importance of the purchasing activity for the companies, by increased use of information technology in the supply chain and by the lack of papers about this subject in Brazil. It aims at understanding the practices of e-procurement in the companies, identifying existing models, the advantages, benefits, critical success factors for implementation and difficulties of its use. In addition, a balance of the publications was performed considering the principal collection in the Web of Science, pointing to the main periods for publications, as well as, the journals on which the papers were published. It is possible to observe tendencies about the researches related to the subject. At the end of this study it was also possible to find important conclusions on the subject, among them are the strategic role of e-procurement, since it changes the way companies are inserted in the supply chain; the current focus on technology purchases of indirect materials, and a set of non-financial benefits. The work also contributes presenting suggestions of subjects to conduct future research on this area.
\end{abstract}

Keywords: E-procurement; Supply chain management; purchasing activities.

\section{E -PROCUREMENT: CONCEPTOS, MODELOS Y FACTORES CRÍTICOS DE ÉXITO}

\section{RESUMEN}

Este trabajo es uma revisión bibliográfica sobre el tema e-procurement y fue motivada por la importancia creciente de la actividad de compras para las empresas, por el aumento de uso de la tecnologia de la informacion em la cadena de suprimentos y por la escazes de trabajos sobre el tema en Brasil. El objetivo fue compreender las prácticas de e-procurement en las empresas, identificando los modelos existentes, las ventajas, benefícios, factores críticos para la implementación y dificultades de su uso. Adicionalmente, fue realizado un analise de las publicaciones sobre el tema em la colección principal de la web of Science, indicando los períodos donde hubierón mas publicaciones y tambien los periódicos que mas tienen artículos relacionados. Luego, fue posible identificar las tendencias sobre las investigaciones en el tema. Al final del trabajo fue tambien posible llegar a las conclusiones importantes sobre la función estratégica de e-procurement, una vez que se modifica la manera como las empresas se incluyen em la cadena de suprimentos. El trabajo todavia contribuye presentando sugerencias de assuntos para condución de investigaciones en la área.

REAd | Porto Alegre - Edição 85 - N 3 - Setembro / Dezembro 2016 - p. 312 - 335 
Palabras Clave: E-procurement; Gestión de la cadena de suprimentos; Compras.

\section{INTRODUÇÃO}

O uso de Tecnologias da Informação e Comunicação (TIC) tem sido apontado na literatura como fator essencial para a melhoria da integração e coordenação das cadeias de suprimentos (HANDFIELD; NICHOLS, 1999; BOWESOX, CLOSS, COOPER, 2007; KETIKIDIS et al., 2010). Tais tecnologias também estão criando oportunidades de repensar os modelos de negócio, processos e relações ao longo de toda a cadeia de suprimento de forma a atingir níveis excepcionais de produtividade (KETIKIDIS et al., 2010).

Concomitantemente, desde o final dos anos 90, diversas tecnologias de comércio eletrônico surgiam com a promessa de revolucionar as práticas de trabalho, ameaçando empresas existentes e potencialmente criando novos modelos de negócio na cadeia de suprimentos (SMART, 2009).

Seguindo o crescimento do uso de comércio eletrônico no mercado business-tobusiness (B2B), houve a adoção globalmente significava do e-procurement (DEVARAJ; VAIDYANATHAN; MISHRA, 2012). Essa adoção não se limita ao setor privado e experiências no setor público também começam a ser relatadas (WALKER; BRAMMER, 2012; COSTA; ARANTES, TAVARES, 2013; VAN GREUNEN; HERSELMAN; VAN NIEKERK, 2010). Além disso, o uso do e-procurement tem sido associado às práticas sustentáveis, especialmente com a redução da geração de resíduo sólido (WALKER; BRAMMER, 2012; COSTA; ARANTES, TAVARES, 2013).

Smart (2009) aponta que a literatura a respeito de e-procurement vem crescendo fortemente desde o fim de 1990, quando artigos tratando do impacto da internet e do comércio eletrônico na gestão da cadeia de suprimentos começaram a ser publicados. Apesar da difusão do e-procurement e do crescente interesse da academia pelo assunto, no Brasil, o eprocurement tem sido pouco abordado na literatura. Uma busca na base de dados SciELO, em dezembro de 2013, revelou apenas duas publicações sobre o assunto: a) Mota e Rodrigues Filho (2011) que pesquisaram o uso do e-procurement na perspectiva da dualidade da tecnologia; e b) Sigulem e Zcchi (2009) que investigaram o impacto da adoção do $e$ procurement nas compras do sistema de organizações de saúde brasileiras.

REAd | Porto Alegre - Edição 85 - N 3 - Setembro / Dezembro 2016 - p. 312 - 335 
Assim, o objetivo deste artigo é realizar um estudo sobre a evolução das publicações de artigos a respeito de e-procurement, nos periódicos da coleção principal da Web os Science, especialmente as áreas de administração, administração de operações, engenharia e ciência da informação ("business economics", "engineering”, "operations research management" e " information science library science"), tendo-se em vista que o $e$ procurement é elemento central para gestão da cadeia de suprimentos. O levantamento buscou compreender as práticas de e-procurement nas empresas, identificando os modelos existentes, as vantagens, benefícios, fatores críticos para a implementação e dificuldades de seu uso. Além disso, busca-se também levantar questões e proposições para trabalhos futuros sobre o tema para a realidade brasileira

Além desta introdução, este artigo está estruturado em 2 partes: A primeira parte traz uma balanço das publicações sobre e-procurement contida na coleção principal da Web of Science, especificamente nas áreas de pesquisa "business economics", "engineering", "operations research management" e " information science library science". Na segunda, é apresentada uma breve revisão da literatura sobre e-procurement, onde são discutidos, principalmente, os conceitos, modelos e usos do e-procurement, os benefícios alcançados e os fatores os fatores de sucesso para a implantação do e-procurement.. Por fim são apresentadas nas considerações finais questões a serem tratadas em pesquisas futuras em organizações brasileiras.

\section{BALANÇO DAS PUBLICAÇÕES SOBRE E-PROCUREMENT NA COLEÇÃO PRINCIPAL DA WEB OS SCIENCE}

Considerando a importância do e-procurement na gestão da cadeia de suprimentos, esta seção traz uma balanço sobre a evolução das publicações a respeito do assunto. Este balanço foi desenvolvido utilizando a plataforma de pesquisa Thomson Reuters - Web of Science, com vistas a verificar a evolução das publicações no período de 2000 à 2015, na Coleção Principal da Web of Science. Utilizou-se na pesquisa básica o descritor " $e$ procurement" e selecionou-se o campo "título". Foram recuperados 231 documentos. Em seguida, selecionou-se as áreas de pesquisa: "business economics", "engineering”, "operations research management" e " information science library science", chegou-se a 158. Desses, os resultados foram limitados a tipo de documento - artigo, sendo que restaram 79. Selecionou-se em seguida, os registros que dispunham textos completos a, assim, chegouREAd | Porto Alegre - Edição 85 - N 3 - Setembro / Dezembro 2016 - p. 312 - 335 
se a 52 artigos. No entanto, nem todos os artigos foram utilizados para compor a base teórica do artigo apresentada na parte 2 deste trabalho.

A escolha artigos publicados na base de dados Web of Science foi feita devido à sua importância na academia e por estar disponível na instituição de ensino ao qual os autores estão vinculados. Além disso, essa base de dados possui um número significativo de periódicos indexados disponíveis e congrega os principais periódicos da área de administração.

Para a análise dos dados, observaram-se o ano de publicação do artigo, o periódico no qual os artigos foram publicados, as áreas de pesquisa da Web of Science contempladas e os temas abordados.

As primeiras publicações sobre e-procurement datam de 2000, conforme pode ser observado no gráfico 1. No entanto, são os anos de 2008, 2009, 2010 e 2014, que apresentam os maiores índices de publicações.

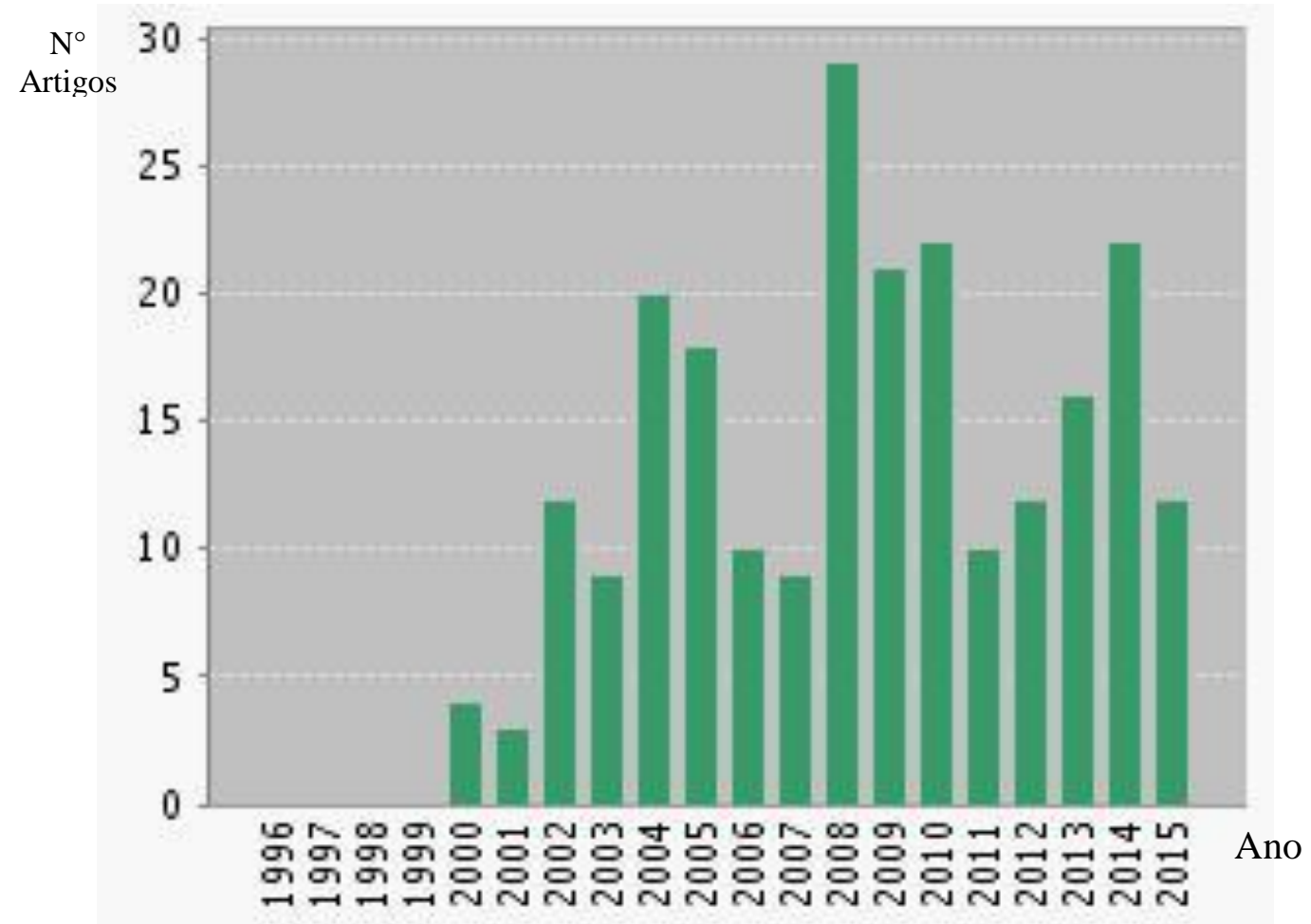

Gráfico 1- Evolução do número de artigos publicados de 2000 a 2015

Fonte: Web of Science

Com relação especificamente à amostra de 52 artigos considerados neste trabalho, a maior concentração de artigos ocorre nos anos de 2004, 2008 e 2010,(Tabela 1). Os artigos concentram-se nas áreas de pesquisa Business \& Economics e Engineering e Operations REAd | Porto Alegre - Edição 85 - N 3 - Setembro / Dezembro 2016 - p. 312 - 335 
Research \& Management Science, o que sugere interesse por parte dos pesquisadores nos aspectos relacionados ao impacto do e-procurement nos negócios e na gestão das empresa (Tabela 1).

Tabela 1- Ano das publicações e áreas de pesquisa

\begin{tabular}{|c|c|c|c|}
\hline Variável & & Número & $\begin{array}{c}\text { Porcentagem } \\
(\%)\end{array}$ \\
\hline Ano de publicação & $\begin{array}{l}2003 \\
2004 \\
2005 \\
2006 \\
2007 \\
2008 \\
2009 \\
2010 \\
2011 \\
2012 \\
2013 \\
2014 \\
2015 \\
\end{array}$ & $\begin{array}{l}2 \\
8 \\
3 \\
3 \\
2 \\
7 \\
2 \\
8 \\
3 \\
5 \\
5 \\
3 \\
1 \\
\end{array}$ & $\begin{array}{c}4 \\
15 \\
6 \\
6 \\
4 \\
13 \\
4 \\
15 \\
6 \\
10 \\
10 \\
6 \\
2 \\
\end{array}$ \\
\hline \multicolumn{4}{|l|}{ Áreas de pesquisa da Web of Science } \\
\hline $\begin{array}{l}\text { Business \& Economics } \\
\text { Operations Research \& Management Science } \\
\text { Engineering } \\
\text { Information Science \& Library Science } \\
\text { Engineering; Operations Research \& Management Sc } \\
\text { Business \& Economics; Operations Research \& Man } \\
\text { Information Science \& Library Science; Business \& } \\
\text { Business \& Economics; Engineering } \\
\text { Engineering; Business \& Economics; Operations Res } \\
\text { Management Science }\end{array}$ & $\begin{array}{l}\text { Science } \\
\text { hics }\end{array}$ & $\begin{array}{c}18 \\
2 \\
3 \\
3 \\
18 \\
2 \\
1 \\
3 \\
2\end{array}$ & $\begin{array}{c}35 \\
4 \\
6 \\
6 \\
35 \\
4 \\
2 \\
6 \\
4\end{array}$ \\
\hline
\end{tabular}

\section{Fonte: os autores}

Com relação ao impacto das publicações, observa-se que o periódico de maior impacto é o Journal of Operations Management, com fator de impacto 7,692- que contém um artigo sobre o assunto. Os periódicos que contem o maior número artigos são o International Journal of Production Economics (fator de impacto 3,069) e o Production Planning \& Control (fator de impacto 1,733). A publicação de menor impacto é o Operations Research Letters (fator de impacto 0,864) (Tabela 2). Ou seja, dos 52 artigos considerados na amostra deste trabalho, 51 são classificados com A1 pela CAPES e 1 como A2, considerando os critérios adotados na Avaliação Trienal 2013 para a área de Administração, Ciências Contábeis e Turismo.

REAd | Porto Alegre - Edição 85 - N 3 - Setembro / Dezembro 2016 - p. 312 - 335 
Tabela 2 - Relação de periódicos e os respectivos fatores de impacto.

\begin{tabular}{|c|c|c|c|}
\hline Periódico & $\begin{array}{c}\mathbf{N}^{\circ} \text { de } \\
\text { Publicações }\end{array}$ & Pais & $\begin{array}{l}\text { Fator de } \\
\text { impacto }\end{array}$ \\
\hline JOURNAL OF OPERATIONS MANAGEMENT & 1 & Holanda & 7,692 \\
\hline $\begin{array}{l}\text { OMEGA-INTERNATIONAL JOURNAL OF } \\
\text { MANAGEMENT SCIENCE }\end{array}$ & 1 & Holanda & 4,139 \\
\hline $\begin{array}{l}\text { SUPPLY CHAIN MANAGEMENT-AN } \\
\text { INTERNATIONAL JOURNAL }\end{array}$ & 4 & Inglaterra & 3,902 \\
\hline TECHNOVATION & 2 & Inglaterra & 3,636 \\
\hline INFORMATION \& MANAGEMENT & 1 & Holanda & 3,105 \\
\hline $\begin{array}{l}\text { INTERNATIONAL JOURNAL OF PRODUCTION } \\
\text { ECONOMICS }\end{array}$ & 6 & Holanda & 3,069 \\
\hline $\begin{array}{l}\text { INTERNATIONAL JOURNAL OF OPERATIONS \& } \\
\text { PRODUCTION MANAGEMENT }\end{array}$ & 3 & Inglaterra & 2,612 \\
\hline EXPERT SYSTEMS WITH APPLICATIONS & 1 & Inglaterra & 2,571 \\
\hline INFORMATION SYSTEMS JOURNAL & 1 & USA & 2,566 \\
\hline DECISION SCIENCES & 1 & USA & 2,56 \\
\hline $\begin{array}{l}\text { JOURNAL OF PURCHASING AND SUPPLY } \\
\text { MANAGEMENT }\end{array}$ & 2 & Inglaterra & 2,521 \\
\hline GOVERNMENT INFORMATION QUARTERLY & 1 & USA & 2,453 \\
\hline $\begin{array}{l}\text { INTERNATIONAL JOURNAL OF INFORMATION } \\
\text { MANAGEMENT }\end{array}$ & 1 & Inglaterra & 2,432 \\
\hline $\begin{array}{l}\text { INTERNATIONAL JOURNAL OF SERVICE INDUSTRY } \\
\text { MANAGEMENT }\end{array}$ & 1 & Inglaterra & 2,402 \\
\hline INDUSTRIAL MARKETING MANAGEMENT & 1 & USA & 2,379 \\
\hline $\begin{array}{l}\text { ELECTRONIC COMMERCE RESEARCH AND } \\
\text { APPLICATIONS }\end{array}$ & 3 & Holanda & 2,34 \\
\hline ADVANCED ENGINEERING INFORMATICS & 1 & Inglaterra & 2,1 \\
\hline INTERNATIONAL JOURNAL OF SYSTEMS SCIENCE & 2 & Inglaterra & 1,859 \\
\hline JCMS-JOURNAL OF COMMON MARKET STUDIES & 1 & EUA & 1,814 \\
\hline $\begin{array}{l}\text { INTERNATIONAL JOURNAL OF PRODUCTION } \\
\text { RESEARCH }\end{array}$ & 4 & Inglaterra & 1,77 \\
\hline PRODUCTION PLANNING \& CONTROL & 6 & Inglaterra & 1,733 \\
\hline GROUP DECISION AND NEGOTIATION & 2 & Holanda & 1,721 \\
\hline IEEE TRANSACTIONS & 1 & USA & 1,582 \\
\hline INDUSTRIAL MANAGEMENT \& DATA SYSTEMS & 1 & Inglaterra & 1,544 \\
\hline $\begin{array}{l}\text { IEEE TRANSACTIONS ON ENGINEERING } \\
\text { MANAGEMENT }\end{array}$ & 3 & USA & 1,526 \\
\hline OPERATIONS RESEARCH LETTERS & 1 & Holanda & 0,864 \\
\hline
\end{tabular}

Fonte: os autores.

As questões abordadas nos artigos são abrangentes (Tabela 3). Há uma concentração maior de trabalhos focalizando metodologias de implantação e avaliação do e-procurement nas empresas, sugerindo a relevância dos processos de implantação e de avaliação para o sucesso a introdução dessa tecnologia. Destaca-se a quantidade de artigos com foco no uso do 
e-procurement no setor governamental, especialmente no que se refere ao impacto na eficiência do setor.

O desenvolvimento de modelos e plataformas, considerando inclusive a interação e o uso de outras TI nas empresa também foram objeto de estudos. Outro tema recorrente é o impacto do e-procurement na atividade de compras, que é diretamente afetada por essa tecnologia, no que se refere ao processo de barganha e ao relacionamento com os fornecedores (Tabela 3).

Tabela 3- Temas abordados.

\begin{tabular}{|l|l|c|l|}
\hline \multicolumn{1}{|c|}{ Tema } & \multicolumn{1}{|c|}{ Assunto Abordado } & \multicolumn{1}{c|}{$\begin{array}{c}\mathbf{N}^{\circ} \text { de } \\
\text { artigos }\end{array}$} & \% \\
\hline $\begin{array}{l}\text { Aplicação no setor } \\
\text { governamental }\end{array}$ & $\begin{array}{l}\text { Desenvolvimento de plataformas; } \\
\text { influencia na eficiência e sustentabilidade } \\
\text { da atividade de compras; restrições para a } \\
\text { adoção }\end{array}$ & 7 & 13 \\
\hline $\begin{array}{l}\text { Aplicação no sistema } \\
\text { logístico e na gestão da } \\
\text { cadeia de suprimentos }\end{array}$ & $\begin{array}{l}\text { Efeito na performance da cadeia e sobre a } \\
\text { flexibilidade de mix e volume }\end{array}$ & 4 & 8 \\
\hline $\begin{array}{l}\text { Modelos de e-procurement } \\
\text { e plataformas }\end{array}$ & $\begin{array}{l}\text { Impacto dos diferentes modelos, uso de } \\
\text { modelos híbridos e diferentes } \\
\text { plataformas, interação com outras TIs }\end{array}$ & 7 & 13 \\
\hline $\begin{array}{l}\text { Impacto nas atividades de } \\
\text { compras }\end{array}$ & $\begin{array}{l}\text { Processo de barganha; organização do } \\
\text { setor; qualidade; segurança da } \\
\text { informação; fatores críticos }\end{array}$ & 7 & 13 \\
\hline $\begin{array}{l}\text { Desenvolvimento de } \\
\text { plataformas }\end{array}$ & $\begin{array}{l}\text { Cloud marketplace; Web based } \text { - } \text { - } \\
\text { procurement, desenvolvimento de } \\
\text { software }\end{array}$ & 3 & 8 \\
\hline Interação com a produção & Planejamento e programação & 2 & 4 \\
\hline Motivação para adoção & Fatores críticos de sucesso; & 5 & 10 \\
\hline Implementação & $\begin{array}{l}\text { Metodologias para implantação e para } \\
\text { avaliação de e resultados e implantação }\end{array}$ & 9 & 17 \\
\hline Aplicação em MPE & Modelos; restrições & 2 & 4 \\
\hline Outros & & 6 & 11 \\
\hline
\end{tabular}

Fonte: os autores.

\section{REVISÃO DA BIBLIOGRAFIA}

Reconhecendo a necessidade de melhor compreender o conceito de e-procurement bem como os seus principais aspectos e limitações da utilização, esta seção traz uma revisão da literatura sobre o tema, com o objetivo de prover uma definição para o termo, apresentar os principais modelos e usos, entender os fatores críticos de sucesso, os benefícios de sua utilização pelas

REAd | Porto Alegre - Edição 85 - N 3 - Setembro / Dezembro 2016 - p. 312 - 335 
empresas, apontar os desafios a serem superados para as organizações usarem de forma eficaz essa tecnologia e apontar caminhos para futuras pesquisas sobre o assunto. A revisão da literatura a seguir, baseou-se em artigos selecionados a partir do balanço apresentado na seção 1 deste trabalho.

\subsection{E-PROCUREMENT: DEFINIÇÃO, MODELOS E USOS}

E-procurement é parte das chamadas tecnologias da informação. As definições são variadas. Presutti (2003, p. 221), por exemplo, define e-procurement como "uma solução tecnológica que facilita as compras corporativas pelo uso da internet". Para Min e Galle (2003), e-procurement diz respeito as transações "business-to business" que utilizam o comércio eletrônico para identificar fontes potenciais de suprimentos, comprar bem e serviços, fazer pagamentos e interagir com fornecedores.

Segundo Wu, Zsidisin e Ross (2007) e-procurement é definido como o uso de tecnologia da informação para facilitar as transações de compras B2B de materiais e serviços. Por sua vez, Angeles e Nath (2007), também definem como sendo compras de produtos e serviços por empresas através da internet. Já, Chang e Wong (2010) definem a adoção de eprocurement como a implementação de tecnologia de pesquisa, cotação e aquisição de bens ou recursos via internet. Por seu turno, Tai, Ho e Wu (2010) têm uma definição mais abrangente. Segundo os autores, e-procurement abrange um escopo maior do que a simples atividade de compra. Envolve atividades estratégicas como fornecimento, negociação com fornecedores e coordenação no desenvolvimento de produtos. Nesse sentido, e-procurement é mais que colocar decisões de compra online. Sua função também inclui a construção de relacionamento entre fornecedores e compradores dentro de uma rede de compra e, além disso, a reestruturação dos processos inter-organizacionais dirigidos por transações.

Nesse sentido, as tecnologias de e-procurement - incluindo softwares, leilões B2B, comércio B2B - focam na automatização de fluxos de trabalho, consolidando e alavancando o poder de compra da organização, e identificando novas oportunidades de fornecimento por meio da internet.

Em suma, quanto a definição, observou-se duas vertentes: autores que propagam $e e$ procurement como ferramenta de suporte à atividade de compras pela internet; e outra, mais

REAd | Porto Alegre - Edição 85 - N 3 - Setembro / Dezembro 2016 - p. 312 - 335 
abrangente, que o considera indutor de mudanças no relacionamento entre compradores e vendedores e no papel da atividade de compras na organização (Quadro 1).

Embora estas definições variem em escopo e detalhes, fica claro que o e-procurement implica no uso de tecnologias de informação e comunicação e de protocolos internet de troca de dados para a obtenção de materiais e serviços pelas empresas.

Quadro 1 - Escopo da aplicação nas organizações e definições.

\begin{tabular}{|l|l|l|}
\hline \multicolumn{2}{|c|}{ Escopo } & \multicolumn{2}{c|}{ Definição } & \multicolumn{1}{c|}{ Autores } \\
\hline \multirow{2}{*}{$\begin{array}{l}\text { Ferramenta de suporte à atividade de } \\
\text { compras pela internet }\end{array}$} & $\begin{array}{l}\text { Solução tecnológica, ou uso de tecnologia da } \\
\text { informação, que facilita as compras } \\
\text { corporativas pelo uso da internet }\end{array}$ & $\begin{array}{l}\text { Pu, Zsidisin e Ross } \\
(2007) \\
\text { Chang e Wong (2010) }\end{array}$ \\
\cline { 2 - 4 } & $\begin{array}{l}\text { Compras de produtos e serviços por } \\
\text { empresas através da internet. }\end{array}$ & $\begin{array}{l}\text { Min e Galle (2003) } \\
\text { Angeles e Nath (2007) }\end{array}$ \\
\hline $\begin{array}{l}\text { Indutor de mudanças no } \\
\text { relacionamento entre compradores e } \\
\text { vendedores e no papel da atividade de } \\
\text { compras na organização }\end{array}$ & $\begin{array}{l}\text { Abrange um escopo maior do que a simples } \\
\text { atividade de compra. Envolve atividades } \\
\text { estratégicas como fornecimento, negociação } \\
\text { com fornecedores e coordenação no } \\
\text { desenvolvimento de produtos. }\end{array}$ & Tai, Ho e Wu (2010) \\
\hline
\end{tabular}

Fonte: os autores.

Cabe salientar que, para Muffatto e Payaro (2004), o e-procurement pode permear todos os estágios do processo de compras, desde a procura por potenciais fornecedores e definição das possíveis alternativas até a concretização da compra, com a diferença de que as atividades são suportadas pela internet, tecnologias de informação e comunicação.

\subsection{Modelos de e-procurement}

Quanto aos modelos de e-procurement, segundo Angeles e Nath (2007), é possível identificar três deles, a saber: i) centrado no fornecedor, ii) centrado no comprador e o iii) $e$ marketplace.

O modelo centrado no fornecedor é adotado por empresas fornecedoras que colocam num portal seu catálogo de produtos e oferecem aos seus clientes a possibilidade de consultar o catálogo, encomendar produtos e serviços, dentre outras funcionalidades. Nesse modelo, cabe a empresa fornecedora o desenvolvimento do portal e a manutenção dos catálogos. Por

REAd | Porto Alegre - Edição 85 - N 3 - Setembro / Dezembro 2016 - p. 312 - 335 
atender ao consumidor final, pode também ser classificado como e-commerce (WU; ZSIDISIN; ROSS, 2007).

Já no modelo centrado no comprador, a entidade compradora reúne num sistema, sob o seu controle e num único catálogo, a informação de múltiplos fornecedores, sobre a qual realiza as suas atividades de e-procurement com a possibilidade de integração com outros sistemas empresariais. Esse modelo permite que a empresa compradora tenha grande controle sobre o sistema, mas é de extrema importância que haja uma ligação próxima com os fornecedores, uma vez que estes precisam oferecer informações a respeito dos produtos, condições de venda e demais informações utilizadas na decisão de compra. O modelo centrado no comprador facilita a integração do e-procurement com o back-office da empresa e não é tão dispendioso, nem para a empresa compradora e nem para o fornecedor (ANGELES; NATH, 2007). Apesar disso, para os fornecedores, o modelo pode não ser o mais vantajoso, pois a empresa fornecedora tem que se relacionar com vários clientes por meio de seus portais. Os fornecedores terão de lidar com vários sistemas diferentes, o que pode não ser muito prático Por fim, o modelo e-marketplace suporta vários clientes e vários fornecedores, na procura de uma relação de "muitos-para-muitos", com soluções exploradas por uma terceira entidade - intermediário -, cujo modelo de negócio passa pela cobrança de um valor de subscrição e de taxas aplicadas às transações. O Quadro 2 resume os principais modelos de e-procurement.

Quadro 2- Principais modelos de e-procurement.

\begin{tabular}{|l|l|l|}
\hline \multicolumn{1}{|c|}{ Modelos } & \multicolumn{1}{c|}{ Características } \\
\hline Centrado no fornecedor & $\begin{array}{l}\text { Empresas fornecedoras colocam num portal seu } \\
\text { catálogo de produtos e oferecem aos seus clientes a } \\
\text { possibilidade de consultar o catálogo, encomendar os } \\
\text { produtos serviços, dentre outras funcionalidades. }\end{array}$ & $\begin{array}{l}\text { Wu, Zsidisin e Ross } \\
(2007)\end{array}$ \\
\hline Centrado no comprador & $\begin{array}{l}\text { Entidade compradora reúne num sistema, sob o seu } \\
\text { controle e num único catálogo, a informação de } \\
\text { múltiplos fornecedores, sobre a qual realiza as suas } \\
\text { atividades de e-procurement, com a possibilidade de } \\
\text { integração com outros sistemas empresariais. }\end{array}$ & $\begin{array}{l}\text { Angeles e Nath, } \\
(2007)\end{array}$ \\
\hline \multirow{2}{*}{ E-marketplace } & $\begin{array}{l}\text { Vários clientes e vários fornecedores, na procura de } \\
\text { uma relação de "muitos-para-muitos", com soluções } \\
\text { exploradas por uma terceira entidade - intermediário. }\end{array}$ & $\begin{array}{l}\text { Angeles e Nath, } \\
(2007)\end{array}$ \\
\hline
\end{tabular}

Fonte: os autores.

\subsection{Produtos transacionados pelos sistemas de e-procurement}

REAd | Porto Alegre - Edição 85 - N 3 - Setembro / Dezembro 2016 - p. 312 - 335 
Quanto aos produtos transacionados, é possível dividi-los em compras diretas e compras indiretas (CHANG; MARKATSORIS; RICHARDS, 2004; TAI; HO; WU, 2010; VAIDYANATHAN; DEVARAJ; D’ARCY, 2012).

- Compras diretas: são as orientadas para a produção e referem-se à compra de matériasprimas e partes necessárias para a fabricação de produtos finais.

- Compras indiretas: são aquelas que não são orientadas para a produção e, geralmente, tratam de suprimentos de manutenção, reparo e operações (MRO).

A compra indireta geralmente não é efetivamente controlado ou automatizado pela maioria das empresas, implicando em maiores custos. Recentemente é possível observar melhorias no uso de e-procurement no processo de aquisição dos produtos de MRO.

\subsection{Usos ou Aplicações do e-procurement pelas organizações}

Autores como Muffatto e Payaro (2002), advogam que o e-procurement se aplica também a compras de produtos de relativo alto valor. Porém, são compras menos frequentes e não estão incluídas nas compras regulares da empresa. Esta categoria, por sua vez, exige que todos os estágios do processo de procurement sejam seguidos. Dado o valor significativamente alto dos produtos, o comprador tem um risco maior envolvido na compra. Dessa forma, deve reunir todas as informações necessárias a respeito dos fornecedores em potencial e dos produtos a serem a adquiridos.

De acordo com Walker e Harland (2008), apesar de os fornecedores de software promoverem o uso do e-procurement como apropriado para compras de todos os tipos de materiais (diretos e indiretos), a tecnologia tem sido usada, principalmente, para compra de um limitado conjunto de produtos, predominantemente suprimentos de escritório e suprimentos de MRO.

Para Gunasekaran et al. (2009), as empresas têm adotado sistemas de e-procurement para compras de (MRO) incluindo itens como materiais de escritório, computadores, solventes de limpeza e móveis de escritório. Isto porque, segundo Tai, Ho e Wu (2010), o uso do e-procurement para compras de materiais diretos envolve muito mais colaboração entre compradores e fornecedores do que a exigida para compra de materiais indiretos, dada a complexidade e peculiaridades de produtos centrais como bens de capital, serviços e mercadorias não padronizadas. As aplicações do e-procurement estão sumarizadas no Quadro 3.

REAd | Porto Alegre - Edição 85 - N 3 - Setembro / Dezembro 2016 - p. 312 - 335 
Quadro 3- Aplicações do e-procurement pelas organizações.

\begin{tabular}{|l|l|}
\hline \multicolumn{2}{|c|}{ Aplicações do e-procurement pelas organizações } \\
\hline Compras de produtos de relativo alto valor & Muffatto e Payaro (2002) \\
\hline $\begin{array}{l}\text { Predominantemente para aquisição suprimentos de escritório e suprimentos } \\
\text { de manutenção, reparo e operações (MRO). }\end{array}$ & Walker e Harland (2008) \\
\hline $\begin{array}{l}\text { Compras de materiais de operação, manutenção e administração (materiais } \\
\text { de escritório, computadores, solventes de limpeza e móveis de escritório) }\end{array}$ & $\begin{array}{l}\text { Gunasekaran et. al (2009); } \\
\text { Walker e Harland (2008) }\end{array}$ \\
\hline
\end{tabular}

Fonte: os autores.

\subsection{Benefícios alcançados pelas organizações com a adoção de e-procurement}

Embora não haja uma metodologia claramente definida para determinar o impacto da adoção do e-procurement nas organizações (RONCHI et al., 2010), vários trabalhos apontam benefícios com a adoção do e-procurement. Esses benefícios podem ser classificados quanto:

- A redução dos custos: refere-se aos custos de aquisição de materiais (order costs) (PRESSUTI, 2003; DAVILA; GUPTA; PALMER, 2003; ANGELES; NATH, 2007).

- Aos objetivos organizacionais: aumento da eficiência das estruturas organizacionais com a redução do tamanho do departamento de compras e dos níveis hierárquicos (VAIDYANATHAN; DEVARAJ, 2008; ANGELES; NATH, 2007).

- À gestão da cadeia de suprimentos: Chang, Tsai e Hsu (2013), apontam como o principal impacto do e-procurement a integração, uma vez que ele facilita o fluxo e o compartilhamento de informação e as atividades de coordenação entre os membros da cadeia de suprimentos. Já autores como Tai, Ho e Wu (2010), advogam que o eprocurement se tornou uma importante ferramenta para o aumento de eficiência de processos interorganizacionais, pois sua adoção não apenas auxilia na atividade de compras em si, mas também implica no estabelecimento de mecanismos de colaboração ao longo da cadeia de suprimentos, especialmente com a melhoria na coordenação e na colaboração entre fornecedores e compradores (Quadro 4). Neste mesmo sentido, Smart (2010) aponta que o e-procurement é uma ferramenta importante para as empresas implementarem suas estratégias de suprimentos. Os benefícios que podem ser alcançados são a redução do número de fornecedores e o aumento do volume de compras em segmentos muito competitivos quanto ao preço.

Outros benefícios relatados na literatura são:

REAd | Porto Alegre - Edição 85 - N 3 - Setembro / Dezembro 2016 - p. 312 - 335 
- controle em tempo real dos gastos internos, comparando-a com o orçamento, de forma a detectar problemas rapidamente (DAVILA; GUPTA; PALMER, 2003; PUSCHMANN, ALT, 2005 )

- transparência interna e nas relações com os fornecedores, quanto às condições contratuais, o tempo e as condições de cada ordem, controle de pedidos e rastreamento, etc. (PUSCHMANN; ALT; 2005; VAIDYANATHAN; DEVARAJ, 2008)

- descentralização, pois permite que os usuários façam pedidos dentro de um contrato pré-negociado, reduzindo, assim, o trabalho de escritório para o departamento de compras e dando maior autonomia para os usuários finais (MUFFATO; PAYARO; 2004; PUSCHMANN; ALT, 2005)

Quadro 4 - Natureza do impacto da implantação do e-procurement.

\begin{tabular}{|l|l|l|l|}
\hline $\begin{array}{l}\text { Natureza do impacto } \\
\text { do e-procurement }\end{array}$ & \multicolumn{1}{c|}{ Dimensão } & \multicolumn{2}{c|}{ Característica } \\
\hline Estratégico & $\begin{array}{l}\text { Relacionamento entre } \\
\text { fornecedores e } \\
\text { compradores }\end{array}$ & $\begin{array}{l}\text { Melhoria na coordenação e na colaboração } \\
\text { entre fornecedores e compradores. }\end{array}$ & Tai, Ho e Wu \\
Operacional & Eficiência & $\begin{array}{l}\text { Melhoria de desempenho de fornecedores } \\
\text { e compradores, processos de integração e } \\
\text { automação. }\end{array}$ & \\
\hline
\end{tabular}

Fonte: os autores.

\subsection{Fatores Críticos de Sucesso na Implementação de e-Procurement nas Organizações}

Vários são os fatores críticos de sucesso elencados na literatura. A seguir destaca-se alguns aspectos baseados nos trabalhos de Puschmann e Alt ( 2005), Angeles e Nath (2007), Davila, et al. (2003) :

- Realinhamento da operação de compras.

- Reorganização geral do processo de compra e outros processos organizacionais afetados pelo e-procurement.

- Preparação de catálogos com a quantidade certa de produtos e com produtos de boa qualidade.

- Participação de fornecedores no estágio inicial.

REAd | Porto Alegre - Edição 85 - N 3 - Setembro / Dezembro 2016 - p. 312 - 335 
- Integração do e-procurement com o sistema back-end (um programa que executa tarefas não diretamente controladas pelo usuário - como o banco de dados - programa que executa tarefas secundárias).

- Gerenciamento de catálogo: refere-se ao conteúdo e a hospedagem, que pode ser na própria empresa, no website do fornecedor ou em marketplaces.

- Escolha do sistema de e-procurement: deve ajustar-se às necessidades específicas verificadas em cada caso. Nesse aspecto, um fator importante a ser observado é a integração do sistema de e-procurement com os outros sistemas relevantes da empresa como o Enterprise Resource Planning (ERP), uma vez que este pode ser fornecido por e outra empresa. Assim, a decisão de qual sistema de e-procurement utilizar pode ser dependente dos sistemas já existentes na organização. Além disso, o sistema de $e$ procurement não deve apenas interagir com os sistemas de informação internos préexistentes, mas deve também cooperar com a parte externa, principalmente com fornecedores e clientes

- Racionalizar o gerenciamento dos fornecedores. Entre as práticas recomendadas está a redução do número de fornecedores com as quais a empresa realiza e-procurement. $\mathrm{O}$ objetivo é consolidar fornecedores e contratos para alcançar redução significativa dos custos de compras e melhores contratos.

- Infraestrutura de informação e da tecnologia de e-procurement. Decisão de qual solução de e-procurement utilizar: centrada no comprador; centrada no vendedor ou marketplace.

O Quadro 5 sumariza uma lista dos Fatores Críticos de Sucesso de sistemas de $e$ procurement encontrados na literatura.

Quadro 5 - Fatores Críticos de Sucesso de sistemas de e-procurement..

\begin{tabular}{|ll|l|}
\hline \multicolumn{1}{|c|}{ Fatores críticos } & \multicolumn{1}{c|}{ Autores } \\
\hline - & \multicolumn{1}{|c|}{ Realinhamento da operação de compras. } & \\
- Reorganização geral do processo de compra. & \\
- Preparação de catálogos com a quantidade certa de produtos e com & \\
- & Produtos de boa qualidade. \\
- Integraçããa do e-procurement com o sistema back-end (um programa & \\
& que executa tarefas não diretamente controladas pelo usuário - como o \\
banco de dados - programa que executa tarefas secundárias). & \\
- Gerenciamento de conteúdos de catálogos & \\
\hline & Racionalizar o gerenciamento dos fornecedores & \\
\hline
\end{tabular}

REAd | Porto Alegre - Edição 85 - N 3 - Setembro / Dezembro 2016 - p. 312 - 335 


\begin{tabular}{|c|c|}
\hline $\begin{array}{l}\text { - } \quad \text { Reengenharia do processo de negócio afetado pelo e-procurement } \\
\text { - Infraestrutura de tecnologia de } e \text {-procurement }\end{array}$ & \\
\hline - $\quad$ Seleção do sistema & $\begin{array}{l}\text { Davila, et al. (2003) } \\
\text { Puschman e Alt (2005) } \\
\text { Angeles e Nath (2007) }\end{array}$ \\
\hline $\begin{array}{ll}\text { - } & \text { Treinamento do usurário } \\
\text { - } & \text { Funcionamento das plataformas de } e \text {-procurement } \\
\text { - } & \text { Resistencia à mudança nas empresas } \\
\text { - } & \text { Profissionais qualificados }\end{array}$ & $\begin{array}{l}\text { Costa, Arantes e } \\
\text { Tavares }(2013)\end{array}$ \\
\hline
\end{tabular}

Fonte: os autores.

\subsection{Adoção de e-procurement em pequenas e médias empresas (PME)}

O e-procurement em PMEs não tem recebido muita atenção por pesquisadores. No entanto, a tecnologia está começando a ser cada vez mais utilizada pelas PMEs, inclusive para melhoria de práticas relativas ao gerenciamento da cadeia de suprimentos em operações domésticas e internacionais. Embora o uso de e-procurement em PMEs tenha sido pouco estudado, é sabido que essas empresas têm um papel muito importante na cadeia de suprimentos e na economia global. Por isso a importância desse tipo de estudo (GUNASEKARAN et al., 2009).

Vitkauskaite e Gatautis (2008) e Renna e Argoneto (2010) advogam que há grande pressão para que as PMEs adotem a tecnologia de e-procurement. Um dos motivos é que nas cadeias de suprimentos, as grande empresas o tem adotado e estão impondo sua utilização aos seus fornecedores. Ocorre que, na maioria das vezes, a maior parte dos fornecedores é composta de PMEs (RENNA; ARGONETO, 2010). Outro aspecto importante, apontado por Vitkauskaite e Gatautis (2008), é que a adoção de tal tecnologia é importante para as PMEs sobreviverem e adaptarem seus negócios no cenário futuro de e-business.

As principais vantagens para as PMEs são: i) acesso ao mercado internacional ao mínimo custo, o que poderia trazer vantagem competitiva para as empresas; ii) redução dos custos de transação; iii) redução dos custos de emissão de ordem de compra de partes e componentes, no contexto de manufatura (RENNA; ARGONETO, 2010). Para Renna e Argoneto (2010) o modelo e-procurement que deve prevalecer entre as PMEs é o de emarketplace, com ênfase nos produtos industriais de MRO.

A adoção de e-procurement coloca grandes desafios para pequenas e médias empresas. Um grande impedimento para a adoção é a falta de consciência a respeito do e-procurement e

REAd | Porto Alegre - Edição 85 - N 3 - Setembro / Dezembro 2016 - p. 312 - 335 
seus impactos no desempenho da organização. A Figura 1 ilustra graficamente o quadro teórico proposto por Gunasekaran et al. (2009) para adoção do e-procurement em PMEs

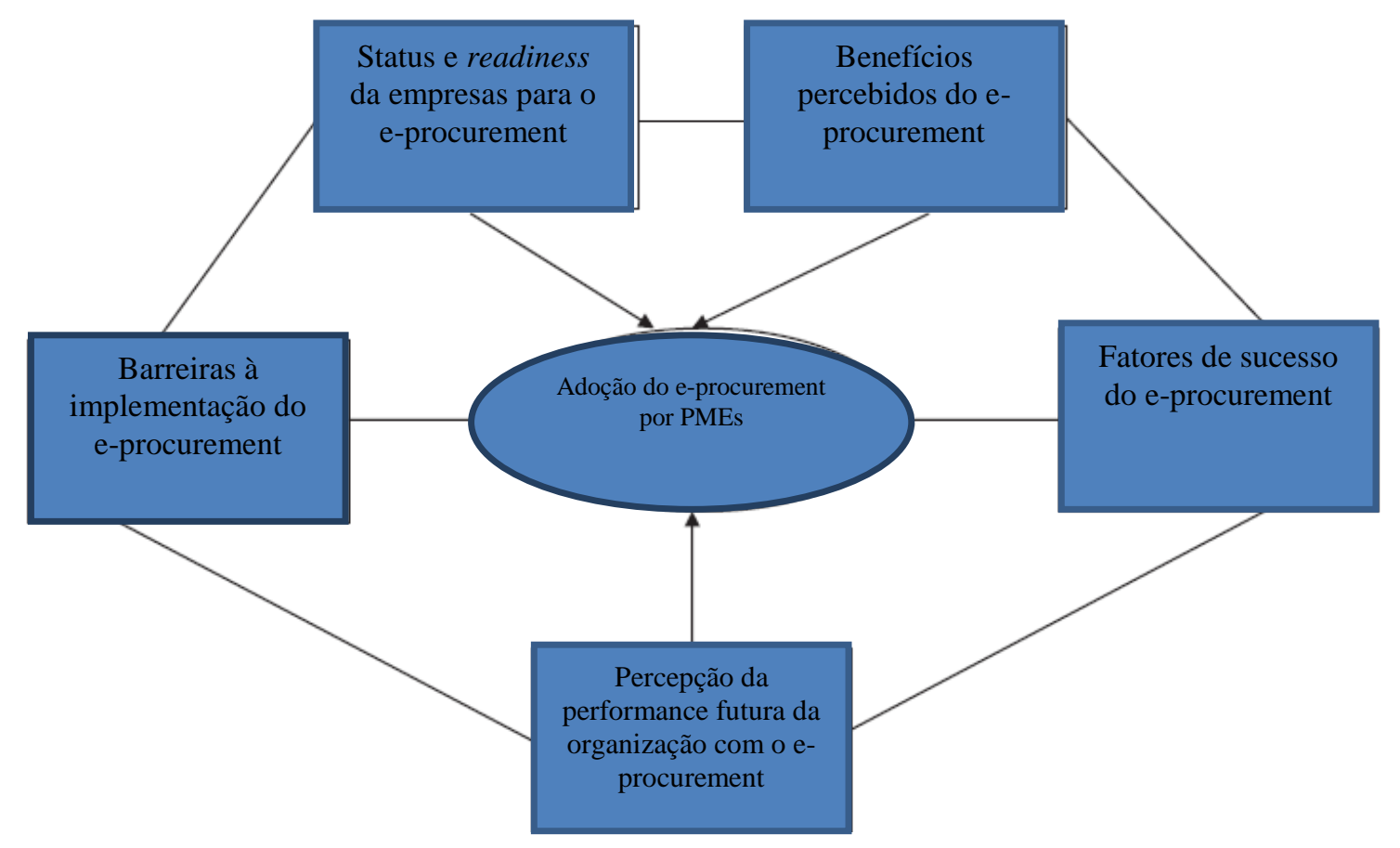

Figura 1 - Quadro teórico de adoção de e-procurement em pequenas e médias empresas.

Fonte: Gunasekaran et al. (2009, p. 163).

- Status e readiness das empresas para o e-procurement: segundo os autores, o sucesso da adoção do e-procurement depende de fatores tecnológicos, comportamentais e organizacionais. Para eles, grandes organizações, inovadoras e com escritórios centralizados são mais propensas a adotar o sistema. São destacadas duas importantes áreas que influenciam a adoção, são elas: aspectos comportamentais e o processo de compras. Dessa forma, conclui-se que as pessoas que já fazem parte da empresa, o processo de compra atual e a tecnologia utilizada, influenciarão sobremaneira a adoção do e-procurement.

- Benefícios percebidos_do e-procurement: o nível de compreensão a respeito dos benefícios trazidos pelo e-procurement irá influenciar sua adoção. Por exemplo, se ninguém na empresa está ciente dos benefícios do sistema (não só os financeiros, mas também os não financeiros), vai haver pouco incentivo para adoção. Apesar dos muitos benefícios decorrentes do uso do e-procurement já elencados na seção 4, Panayiotou et al. (2004) afirmam que muitas empresas enxergam o sistema apenas como o uso de TI para a troca de informação com fornecedores, mas falham em não REAd | Porto Alegre - Edição 85 - N 3 - Setembro / Dezembro 2016 - p. 312 - 335 
ver os benefícios estratégicos e táticos. Essa falta de entendimento pode desencorajar a adoção.

- Barreiras ao e-procurement: de acordo com Hawking et al. (2004), barreiras como a falta de capital, de perícia e habilidades técnicas, de tecnologias e ferramentas, a resistência à mudança e a falta de suporte gerencial poderá atrapalhar a adoção do $e$ procurement e influenciar negativamente seu desempenho após a implementação.

- Fatores críticos de sucesso do e-procurement em PMEs: esse tipo de empresa tende a ser flexível e inovadora. No entanto tem limitações em relação ao capital e ao conhecimento no que diz respeito a novas práticas e tecnologias. Considerando essas características, é necessário que sejam desenvolvidas estratégias, táticas e políticas operacionais apropriadas para a implementação do e-procurement em PMEs. O escopo de negócio em termos de volume, mix de produtos, natureza dos produtos (manufaturados ou serviços), e o papel da empresa da cadeia de suprimentos irá influenciar na adoção. Os fatores críticos aqui são: promoção de incentivos, comprometimento da alta gerência, implementação passo a passo (step-by-step) e mecanismos de manutenção e operação do sistema.

- Desempenho organizacional pretendido com o e-procuremet: a maneira como os proprietário e gerentes das PME's acreditam que o e-procurement pode impactar positivamente a empresa, trazendo melhorias de desempenho, vai influenciar a predisposição dos mesmos em relação a adoção. É importante que eles estejam cientes do impacto potencial do e-procurement na performance de curto e longo prazo em áreas como custo de produção/serviços e competitividade. Eles devem reconhecer também o papel do sistema nas alianças estratégicas com clientes e fornecedores. PMEs tendem em focar em performances financeiras mensuráveis em curto prazo, mas não nas de logo prazo. Essa orientação não é desejável no ambiente atual de negócios. Desempenhos de curto são tão importantes como os de longo prazo, o mesmo ocorre em relação ao desempenho financeiro e ao não financeiro, ambos tem grande importância.

\subsection{DESAFIOS PARA A IMPLEMENTAÇÃO DO E-PROCUREMENT}

Angels e Nath (2007), elencam um conjunto dos principais desafios para a implementação do e-procurement:

REAd | Porto Alegre - Edição 85 - No 3 - Setembro / Dezembro 2016 - p. 312 - 335 
- Imaturidade dos serviços de marketplace. Os marketplaces menos desenvolvidos podem não oferecer a linha completa de serviços como orçamentos eletrônicos, leilões reversos ou tradicionais, catálogos eletrônicos e manutenção de um bom número de firmas assinantes.

- Imaturidade dos fornecedores. As empresas devem lidar com a imaturidade tecnológica de seus fornecedores que por vezes não estão preparados para responder às iniciativas de compras eletrônicas. Neste ponto, o tamanho da empresa parece ser um fator importante. Pequenas empresas geralmente são avessas à inovação e tendem a não contar com os conhecimentos técnicos e com a estrutura de TI necessários para responder as requisições de compra eletrônica.

- Imaturidade dos serviços de consultoria. Peculiaridades das diferentes indústrias complicam a situação ainda mais. Os exemplos de grandes empresas são, frequentemente, usados por consultores. Contudo, no caso da implementação em empresas menores, espera-se uma consultoria mais direta e que acompanhe mais de perto o processo de implementação.

- Precificação. Os modelos de precificação muitas vezes são ruins, tanto em relação às licenças para as ferramentas de compra como às taxas de transação nos marketplaces.

- Imaturidade dos próprios softwares de e-procurement. Existem muitos relatos de usuários insatisfeitos com as aplicações da tecnologia de e-procurement. Alguns sistemas são incapazes de lidar com formatos de dados utilizados por grandes fornecedores de software, além disso, não permitem a integração com os sistemas já existentes na empresa.

Um bom número de softwares não é capaz de gerenciar diferenças de jurisdição, moedas correntes e estruturas fiscais de diferentes países, além de não possuir bons mecanismos de autenticação e segurança. Também pode haver outros desafios no que concerne a imaturidade dos softwares quanto às necessidades específicas de cada empresa.

- Falta de formatos padrão para troca de informações. Nesse sentido percebe-se a falta de padronização de códigos referentes a informações de produtos em diferentes catálogos e dos formatos dos próprios catálogos.

- Resistência dos usuários internos da empresa em adotar o sistema de e-procurement. Os funcionários geralmente resistem em aprender sistemas de compras novos, especialmente se eles tiverem meios alternativos para fazerem as referidas compras, REAd | Porto Alegre - Edição 85 - N 3 - Setembro / Dezembro 2016 - p. 312 - 335 
por meio de cartões de compra e submissão de despesas, por exemplo. Inclusive, em relação aos meios antigos para realização das compras, eles não podem deixar de ser usados, uma vez que são necessários para compras de produtos de fornecedores que não usam a tecnologia de comercio eletrônico.

- Custos envolvidos com a implementação. As empresas devem ter cuidado ao calcular os custos decorrentes das iniciativas necessárias para a implantação do sistema. Inicialmente alguns custos não são considerados a princípio e podem acabar excedendo as expectativas. Exemplos de custos: implementação (serviços de consultoria), integração (sistema de gerenciamento de recursos humanos, gerenciamento de ativos de equipamentos, gerenciamento de ativos de TI e financeiros), treinamento dos usuários, reengenharia do processo de compras, custos com licenças, administração (requisições, pedidos, processos de pagamento). Especial atenção deve ser dada aos catálogos eletrônicos, pois estes representam uma parcela considerável dos custos.

- Dificuldade de se eliminar o chamado "Maverick Buying”. O termo se refere às compras realizadas sem o uso de processos formais definidos e por pessoas que não estão formalmente autorizadas. Esse tipo de compra é prejudicial para a organização na medida em que dificulta a coleta de dados a respeito dos gastos de cada funcionário, faz com que a visibilidade e controle sobre despesas sejam debilitados, e aumenta os custos com compras na empresa em cerca de $20 \%$.

\section{CONSIDERAÇÕES FINAIS}

Num ambiente mundial de grandes mudanças, sobretudo em relação a novas tecnologias de informação, os processos de negócios das empresas são impactados e tendem a agregar novas ferramentas e aplicações, como é o caso do uso da tecnologia de $e$ procurement.

Diante da escassez de estudos sobre $e$-procurement no Brasil, este trabalho fez um levantamento das publicações sobre o assunto na coleção principal da Web of Science, no período de 2000 a 2015, com o propósito de identificar os principais aspectos tratados sobre o assunto.

Baseando-se nos resultados, esse artigo trouxe primeiramente uma breve revisão sobre e-procurement, desde as definições existentes até os problemas com a implantação e os

REAd | Porto Alegre - Edição 85 - N 3 - Setembro / Dezembro 2016 - p. 312 - 335 
desafios para as empresas. Em seguida apresentou um levantamento dos principais temas abordados pelos artigos no período e também sobre os principais periódicos onde eles estão sendo publicados.

De maneira geral, constatou-se que essa tecnologia pode trazer vários benefícios às empresas usuárias, não só em relação à diminuição dos custos envolvidos no processo de compra (compra de produtos por preços menores, diminuição de redundâncias, redução dos gastos com burocracia no processo, necessidade de um número menor de funcionários envolvidos etc..), como também no que diz respeito ao relacionamento com os outros integrantes da cadeia de suprimentos, permitindo maior coordenação e cooperação entre eles. No entanto, um aspecto a ser destacado, e que tem impacto na cadeia de suprimentos, são os problemas que podem ser percebidos durante a implementação, como a imaturidade tecnológica da empresa e de seus fornecedores.

Dessa forma, esse trabalho contribuir como fonte de informações para revisões bibliográficas e como indicação para outras pesquisas a respeito do assunto, uma vez que elenca um bom número de artigos relevantes publicados sobre e-procurement. Para as empresas, este trabalho serve como um guia para que se pondere sobre os modelos existentes, os desafios envolvidos na implantação e os benefícios que podem ser trazidos pelo uso do $e$ procurement.

Por fim, o balanço das publicações sobre e-procurement, feito na segunda parte desse trabalho, lançou luz sobre como essa tecnologia tem sido investigada e quais os principais aspectos a ela relacionados que estão sendo objeto de pesquisa. Assim, para o contexto brasileiro, é possível elencar uma série de questões a serem investigadas, em trabalhos futuros, nas organizações públicas e privadas brasileiras, tais como:

- De que forma as empresas que fazem uso da tecnologia de e-procurement vencem a questão de falta de maturidade tecnológica da parte dos fornecedores? Deixam de realizar transações com estes fornecedores ou procuram incentivar, de alguma forma, o desenvolvimento tecnológico dos demais membros da cadeia de suprimentos?

- Em empresas quem mantêm o sistema de compras via e-procurement e, além dele, o sistema convencional, como se dá a coordenação entre os dois sistemas?

- Atualmente, no cenário global, qual o estágio de evolução do uso do e-procurement? Em que setores e/ou indústrias a tecnologia é mais presente? Qual parcela das compras REAd | Porto Alegre - Edição 85 - N 3 - Setembro / Dezembro 2016 - p. 312 - 335 
é realizada via e-procurement? Quais os tipos de materiais mais comprados? Ainda prevalece o uso da tecnologia para compra de materiais indiretos?

- Como os fornecedores de software se posicionam? Oferecem opções padronizadas ou personalizadas de software? Como é feita a comunicação entre softwares diferentes, tanto internamente como entre empresas distintas? Quais são as dificuldades técnicas envolvidas?

- Qual modelo de e-procurement mais utilizados em empresas brasileiras?

- Quais são os fatores limitadores para o uso do e-procurement nas empresas brasileiras?

- Quais são os benefícios econômicos e organizacionais obtidos com o uso de $e$ procurement nas empresas brasileira

\section{REFERÊNCIAS}

ANGELES, R.; NATH, R. Business-to-business e-procurement: success factors and challenges to implementation. Supply Chain Management - An International Journal, Surabaya, v. 12, n. 2, p. 104-115, 2007.

BOWERSOX, D. J.; CLOSS, D. J.; COOPER, M. B. Gestão da Cadeia de Suprimentos e Logística. 2. ed. Rio de Janeiro: Elsevier, 2007.

CHANG, H. H.; TSAI, Y. C.; HSU, C. H. E-procurement and supply chain performance. Supply Chain Management- An International Journal, Subaraya, v 18, n. 1, p. 34-51, 2013.

CHANG, H. H.; WONG, K. H. Adoption of e-procurement and participation of e-marketplace on firma performance: Trust as a moderator. Information \& Management, Taiwan, v. 47, n. 56, p. 262-270, 2010.

CHANG, Y; MARKATSORIS, H; RICHARDS, H. Design and implementation of an eprocurement system. Production Planning \& Control, Boca Raton, v. 15, n. 7, p. 634-646, 2004.

COSTA, A. A.; ARANTES, A.; TAVARES, L. V. Evidence of the impacts of public eprocurement: The Portuguese experience. Journal of Purchasing and Supply Management, Amsterdam, v. 19, n. 4, p. 238-246, 2013.

DAVILA, A; GUPTA, M; PALMER, R. Moving Procurement Systems to the Internet: The Adoption and Use of E-procurement Technology Models. European Management Journal, Amsterdam, v. 21, n. 1, p. 11-23, 2003.

REAd | Porto Alegre - Edição 85 - N 3 - Setembro / Dezembro 2016 - p. 312 - 335 
DEVARAJ, S; VAIDYANATHAN, G.; MISHRA, A. N. Effect of purchase volume flexibility and purchase mix flexibility on e-procurement performance: an analysis of two perspectives. Journal of Operations Management, Amsterdam, v. 30, n. 7-8, p. 509-520, 2012.

GUNASEKARAN, A.; McGAUGHEY, R. E; NGAI, E. W. T.; RAI, B. K. et al. E-procurement adoption in the Southcoast SME's. International Journal of Production Economics, Hong Kong, v. 122, n. 1, p. 161-175, 2008.

HANDFIELD, R.B.; NICHOLS E.L. Introduction to Supply Chain Management. Upper Saddle River, NJ: Prentice Hall, 1999.

HAWKING, P., STEIN, A., WYLD, D.C., FOSTER, S. E-procurement: Is the ugly duckling actually a swan down under? Asia Pacific Journal of Marketing and Logistics, Bingley, v. 16, n. 1, p. 3-26, 2004.

KETIKIDIS, P.H.; KONTOGEORGIS A.; STALIDIS G. e KAGGELIDES, K. Applying: eprocurement system in the healthcare: the EPOS paradigm. International Journal of Systems Science, Boca Raton, v. 41, n. 3, p. 281-299, 2010.

MOTA, F. P.B.; RODRIGUES FILHO, J. Public e-procurement and the duality of technology: a comparative study in the context of Brazil and of the state of Paraíba. Journal of Information Systems and Technology Management, São Paulo, v. 8, n. 2, p. 315-330, 2011.

MUFFATTO, M.; PAYARO, A. Implementation of e-procurement and e-fulfillment processes: A comparison of cases in the motorcycle industry. International Journal of Production Economics, Amsterdam, v. 89, n. 3, p. 338-351, 2004.

PRESUTTI, W. JR. Supply management and e-procurement: creating value added in the supply chain. Industrial Marketing Management, Amsterdam, v. 32, n .3, p. 219- 226, 2003.

PUSCHMANN, T.; ALT, R. Successful use of e-procurement in supply chains. Supply Chain Management - An International Journal, Amsterdam, v. 10, n. 2, p. 122-133, 2005.

RENNA, P.; ARGONETO, P. Production planning and automated negotiation for SMEs: An agent based e-procurement application. International Journal of Production Economics, Amsterdam, v. 127, n. 1, p. 73-84, 2010.

RONCHI, S. et al. What is the value of an IT e-procurement system? Journal of Purchasing and Supply Management, Amsterdam, v. 16, n. 2, p. 131-140, 2010.

SAMPIERI, R. H.; COLLADO, C. F.; LUCIO, P. B. Metodologia de Pesquisa. 3. ed. São Paulo: McGraw, 2006.

SIGULEM, F.; ZUCCHI, P. E-procurement in the Brazilian healthcare system: the impact of joint drug purchases by a hospital network. Revista Panamericana de Salud Publica-Pan American Journal of Public Health, Washington, v. 26, n. 5, p. 429-434, 2009.

REAd | Porto Alegre - Edição 85 - N 3 - Setembro / Dezembro 2016 - p. 312 - 335 
SMART, A. Exploring the business case for e-procurement. International Journal of Physical Distribution \& Logistics Management, Bingley, v. 40, n. 3, p. 181-201, 2010.

TAI, Y. M.; HO, C. F.; WU, W. H. The performance impact of implementing Web-based eprocurement systems. International Journal of Production Research,Abingdon, v. 48, n. 18, p. 5397-5414, 2010.

VAIDYANATHAN, G.; DEVARAJ, S.; D'ARCY, J. Does Security Impact E-procurement Performance? Testing a Model of Direct and Moderated Effects. Decision Sciences, Hoboken, v 43, n. 3, p 437-458, 2012.

VAIDYANATHAN. G; DEVARAJ, S. The role of quality in e-procurement performance: An empirical analysis. Journal of Operations Management, Amsterdam, v. 26, n. 3, p. 407-425, 2008.

VAN GREUNEN, D; HERSELMAN, M. E. VAN NIEKERK, J. Implementation of regulationbased e-procurement in the Eastern Cape provincial administration. African Journal of Business Management, [s.1.]v. 4, n. 17, p. 3655-3665, 2010.

VITKAUSKAITE, E.; GATAUTIS, R. E-procurement perspectives in construction sector smes. Journal of Civil Engineering and Management, Abingdon, v. 14, n. 4, p. 287-294, 2008.

WALKER, H.; BRAMMER, S. The relationship between sustainable procurement and eprocurement in the public sector. International Journal of Production Economics, Amsterdam, v. 140, n. 1, p. 256-268, 2012.

WALKER, H.; HARLAND C. E-procurement in the United Nations: influences, issues and impact. International Journal of Operations \& Production Management, Bingley, v. 28, n. 9-10, p. 831-857, 2008.

WU, F.; ZSIDISIN, G. A.; ROSS, A. D Antecedents and outcomes of e-procurement adoption: An integrative model. Transactions on Engineering Management, [s.1.], v. 54, n. 3, p. 576587, 2007. 